\title{
Moufang Plane and Octonionic Quantum Mechanics`
}

\author{
M. Günaydin ${ }^{1}$, C. Piron ${ }^{2}$, and H. Ruegg ${ }^{2}$ \\ ${ }_{1}$ Physikalisches Institut, Universität Bonn, D-5300 Bonn 1, Federal Republic of Germany \\ 2 Département de Physique Théorique, Université de Genève, CH-1211 Genève 4, Switzerland
}

\begin{abstract}
It is shown that the usual axioms of one-particle Quantum Mechanics can be implemented with projection operators belonging to the exceptional Jordan algebra $J_{3}^{8}$ over real octonions. Certain lemmas on these projection operators are proved by elementary means. Use is made of the Moufang projective plane. It is shown that this plane can be orthocomplemented and that there exists a unique probability function. The result of successive, compatible experiments is shown not to depend on the order in which they are performed, in spite of the non-associativity of octonion multiplication. The algebra of observables and the action of the exceptional group $F_{4}$ is studied, as well as a possible relation with the color group SU(3) and quark confinement.
\end{abstract}

\section{Introduction}

Following Jordan's formulation [1] of Quantum Mechanics in terms of anticommutators, Jordan, von Neumann and Wigner (JNW) [2] showed that all realizations of Quantum Mechanics in terms of anti-commutators (Jordan product) are equivalent to realizations in terms of commutators (Lie product) except in the case of $3 \times 3$ Hermitian octonionic matrices which form the exceptional Jordan algebra. Since this one possible generalization via the Jordan formulation was found too narrow for explaining the then observed nuclear phenomena this approach was abandoned. However, Jordan algebras have since been extensively studied by the mathematicians who established some deep connections between the exceptional Lie groups and the exceptional Jordan algebra $[3,4]$.

After the sixties octonions made their appearance in physics again. Pais, Gamba and others tried to relate the octonions to various internal symmetry schemes in elementary particle physics [5, 6] Goldstine, Horwitz, and Biedenharn studied a Clifford algebra made of octonionic multiplication operators [7].

Günaydin and Gürsey suggested the extension of the underlying field of Quantum Mechanics from complex numbers to the octonion algebra in order to

* Work supported by the Swiss National Science Foundation 
explain the charge space properties of elementary particles on a fundamental level [8-13]. They showed that space-time groups like the Poincaré group can be unitarily implemented on an octonionic space with complex scalar products. The space-time symmetries and quantum mechanical considerations then select out an SU(3) subgroup of the automorphism group $G_{2}$ of octonions as an intrinsic exact symmetry group of the space, which they identified with the color SU(3) group of Gell-Mann and Fritzsch [14]. By taking octonionic quark fields, making the ansatz that the non-associative and non-commutative components of the space be unobservable, they could give an algebraic explanation of the observability of color singlet states only, and the unobservability of quarks and other colored states.

In this paper we answer the following question: is it possible to implement the usual axioms of quantum mechanics with objects belonging to a Jordan algebra? It turns out that this is not possible with the usual Hilbert space formulation of Quantum Mechanics, because the octonion algebra is non associative. So one has to go to a more abstract level [15], starting with the concept of proposition (yes-no experiment). Propositions correspond in the usual case to projection operators, and the structure of the propositional system of Quantum Mechanics is equivalent to the structure of an orthocomplemented projective geometry. At this point one finds the link with octonions. Ruth Moufang [16] has constructed a projective plane coordinated by octonions, and which turns out to be non-Desarguian.

Our aim in this paper is to study the quantum mechanical properties of this non-Desarguian geometry by using Jordan's formulation of it in terms of the exceptional Jordan algebra $[17,18]$. We show that it can be orthocomplemented and that there exists a unique probability function satisfying Gleason's axioms. Successive compatible experiments yield a result which is independent of the order in which they are performed. Hence, on the one-particle level all the axioms of Quantum Mechanics are fullfilled. We further study the action of the automorphism group of the Moufang plane on states and the structure of the algebra of observables.

Physical interpretation of the octonionic Quantum Mechanics poses many problems. An important problem has to do with the possible product states, which is crucial for the algebraic explanation of the unobservability of colored states. Main-difficulty stems from the fact that all irreducible projective geometries of dimension greater than two are Desarguian. One possible way out is to have an imbedding of Desarguian subgeometries. Another possibility is to imbed this projective geometry into a non-irreducible higher geometry. In any case, we hope that the study of these problems may shed further light into the problem of unobservability of colored states.

Before we begin our study of the octonion Quantum Mechanics we should stress the fact that the mathematics underlying our work has long been thoroughly developed by the mathematicians. The lemmas we use or prove may be found in the mathematical literature in some form or other. Our purpose is to reformulate these results in a physicists' language and lay the ground for future work on the subject. 


\section{Octonions and the Exceptional Jordan Algebra}

Real octonion algebra $\mathbb{D}$ is an 8-dimensional division algebra whose elements can in general be decomposed as

$$
O=r_{0} e_{0}+\sum_{A=1}^{7} r_{A} e_{A},
$$

where $r_{0}$ and $r_{A}$ are real numbers, $e_{0}$ is the identity element and $e_{A}$ are the seven imaginary units which obey the multiplication rule

$$
e_{A} e_{B}=-\delta_{A B}+\sum_{C=1}^{7} \eta_{A B C} e_{C} .
$$

$\eta_{A B C}$ are totally anti-symmetric and their non-vanishing components, apart from permutations, are [9]

$\eta_{123}=\eta_{246}=\eta_{435}=\eta_{367}=\eta_{651}=\eta_{572}=\eta_{471}=1$.

The octonion algebra is not associative, but it satisfies a weaker property, namely alternativity, which implies that the associator $\left(\mathrm{O}_{1}, \mathrm{O}_{2}, \mathrm{O}_{3}\right)$ of any 3 octonions is an alternating function of its arguments:

$$
\left(O_{1}, O_{2}, O_{3}\right) \equiv\left(O_{1} O_{2}\right) O_{3}-O_{1}\left(O_{2} O_{3}\right)=\left(O_{3}, O_{1}, O_{2}\right)=-\left(O_{2}, O_{1}, O_{3}\right) .
$$

As a consequence of this property one has

$$
\left(O_{1} O_{1}\right) O_{2}=O_{1}\left(O_{1} O_{2}\right) ; \quad\left(O_{1} O_{2}\right) O_{2}=O_{1}\left(O_{2} O_{2}\right)
$$

and the Moufang identity

$$
\left(O_{1} O_{2}\right)\left(O_{3} O_{1}\right)=O_{1}\left(O_{2} O_{3}\right) O_{1} \text {. }
$$

The conjugate octonion is defined by

$$
\bar{O}=r_{0} e_{0}-\sum_{A=1}^{7} r_{A} e_{A}
$$

and the real bilinear product by [19]

$$
\left(O_{1}, O_{2}\right) \equiv \frac{1}{2}\left(\bar{O}_{1} O_{2}+\bar{O}_{2} O_{1}\right)
$$

which induces the usual octonionic norm

$$
\begin{aligned}
& n(O)=(O, O)=\bar{O} O=O \bar{O} \\
& n\left(O_{1} O_{2}\right)=n\left(O_{1}\right) n\left(O_{2}\right)
\end{aligned}
$$

and has the invariance group $O(8)$.

The real exceptional Jordan algebra $J_{3}^{8}$ is a Jordan algebra whose elements are the $3 \times 3$ hermitian real octonionic matrices

$$
J=\left(\begin{array}{lll}
\alpha_{1} & O_{3} & \bar{O}_{2} \\
\bar{O}_{3} & \alpha_{2} & O_{1} \\
O_{2} & \bar{O}_{1} & \alpha_{3}
\end{array}\right),
$$


where $\alpha_{i}$ are real numbers and $O_{i}$ are real octonions. These matrices form an intrinsically non-associative algebra under the Jordan product

$$
J_{1} \circ J_{2}=\frac{1}{2}\left(J_{1} J_{2}+J_{2} J_{1}\right),
$$

where $J_{1} J_{2}$ is the usual matrix product, and satisfy the Jordan identity:

$$
\left(J_{1} \circ J_{2}\right) \circ J_{1}^{2}=J_{1} \circ\left(J_{2} \circ J_{1}^{2}\right) \text {. }
$$

In the classic work of Jordan et al. [2], it was shown that the algebra $J_{3}^{8}$ is the only Jordan algebra which has no realizations in terms of associative matrices. A onedimensional projection operator $P$ is a Jordan matrix which satisfies the conditions

$$
P^{2}=P \circ P=P ; \quad \operatorname{tr} P=1 .
$$

Any one-dimensional projection operator $P$ belonging to $J_{3}^{8}$ can be brought to the following form [17]

$$
P=\left(\begin{array}{l}
a \\
b \\
c
\end{array}\right)^{(\bar{a} \bar{b} \bar{c})}=\left(\begin{array}{lll}
a \bar{a} & a \bar{b} & a \bar{c} \\
b \bar{a} & b \bar{b} & b \bar{c} \\
c \bar{a} & c \bar{b} & c \bar{c}
\end{array}\right)
$$

where $a, b, c$ are octonions, one of them being pure real, and satisfy

$$
a \bar{a}+b \bar{b}+c \bar{c}=\operatorname{tr} P=1 .
$$

Following Freudenthal [20] one can define the following $X$ product among the Jordan matrices

$$
J_{1} \times J_{2} \equiv J_{1} \circ J_{2}-\frac{1}{2} J_{1} \operatorname{tr} J_{2}-\frac{1}{2} J_{2} \operatorname{tr} J_{1}+\frac{1}{2} I\left(\operatorname{tr} J_{1} \operatorname{tr} J_{2}-\operatorname{tr} J_{1} J_{2}\right),
$$

where $I$ is the $3 \times 3$ identity matrix.

For Jordan matrices on real octonions one has the property that if $J \times J=0$ then $J$ is a scalar multiple of a projection operator i.e.

$$
J \times J=0 \Rightarrow J^{2}=\lambda J ; \quad \operatorname{tr} J=\lambda,
$$

where $\lambda$ is a non-zero real number if $J \neq 0$.

Using the $X$ product one can define a completely symmetric trilinear form:

$$
\left[J_{1}, J_{2}, J_{3}\right] \equiv \operatorname{tr}\left(J_{1} \times J_{2}\right) \circ J_{3} \equiv\left(J_{1} \times J_{2}, J_{3}\right)
$$

which induces the determinental form i.e.

$$
\operatorname{det} J=\frac{1}{3}[J, J, J]=\frac{1}{3} \operatorname{tr} J^{3}-\frac{1}{2} \operatorname{tr} J^{2} \operatorname{tr} J+\frac{1}{6}(\operatorname{tr} J)^{3} .
$$

\section{Group Theoretic Properties of $J_{3}^{8}$}

The exceptional Jordan algebra has, as the automorphism group, the exceptional group $F_{4}$ [4]. The 52 infinitesimal anti-hermitian generators $D$ of $F_{4}$ act as derivations of $J_{3}^{8}$ i.e. they satisfy the property:

$$
D\left(J_{1} \circ J_{2}\right)=\left(D J_{1}\right) \circ J_{2}+J_{1} \circ\left(D J_{2}\right)
$$


for any elements $J_{1}, J_{2}$ of $J_{3}^{8}$. This implies that the derivations annihilate the identity matrix :

$$
D I=0 \text {. }
$$

The 26 traceless Jordan matrices form the basis vectors for an irreducible representation of $F_{4} . F_{4}$ can also be uniquely characterized as the simultaneous invariance group of the quadratic and cubic forms of $J_{3}^{8}$ i.e. as the invariance group of

$$
\left(J_{1}, J_{2}\right)=\operatorname{tr} J_{1} \circ J_{2}
$$

and of

$$
\left[J_{1}, J_{2}, J_{3}\right]=\operatorname{tr}\left(J_{1} \times J_{2}\right) \circ J_{3}
$$

The derivations can be represented by the action of two traceless Jordan matrices $X, Y$ on $J_{3}^{8}$ :

$$
D_{X, Y} J=(X, J, Y)=-(Y, J, X),
$$

where the RHS is the associator

$$
(X, J, Y) \equiv(X \circ J) \circ Y-X \circ(J \circ Y) .
$$

The derivations satisfy the following commutation relations:

$$
\left[D_{X, Y}, D_{Z, W}\right]=D_{D_{X, Y} Z, W}+D_{Z, D_{X, Y} W} .
$$

The automorphisms of the $2 \times 2$ Jordan matrices form an $\mathrm{SO}(9)$ subgroup of $F_{4}$. There are three of them in $F_{4}$. If we denote the elements $X, Y$ of $J_{3}^{8}$ as

$$
X=\left(\begin{array}{lll}
\alpha_{1} & x_{3} & \bar{x}_{2} \\
\bar{x}_{3} & \alpha_{2} & x_{1} \\
x_{2} & \bar{x}_{1} & \alpha_{3}
\end{array}\right)=\sum_{i=1}^{3} \alpha_{i} E_{i}+\left(x_{3}\right)_{12}+\left(x_{2}\right)_{31}+\left(x_{1}\right)_{23}
$$

then the generators of the $\mathrm{SO}(9)$ subgroup leaving the indempotent $E_{3}$ invariant are given by

$$
D_{\left(x_{3}\right)_{12},\left(y_{3}\right)_{12}}, \quad D_{\left(x_{3}\right)_{12}, E_{1}-E_{2}} .
$$

The other $\mathrm{SO}(9)$ subgroups' generators can be obtained by a cyclic permutation of $1,2,3$.

Each $\mathrm{SO}(9)$ subgroup has an $\mathrm{SO}(8)$ subgroup that leaves the diagonal terms invariant. However, the $3 \mathrm{SO}(8)$ subgroups' actions are not independent of each other. These 3 actions are related via the principle of triality [21]. The principle of triality states that given an action $d_{1}$ of $S O(8)$ on the octonion algebra (D) then there exist actions $d_{2}$ and $d_{3}$, unique up to a sign, which satisfy the property

$$
\left(d_{1} O_{1}\right)\left(d_{2} O_{2}\right)=\overline{d_{3} \overline{\left(O_{1} O_{2}\right)}} \forall O_{1}, O_{2} \in \mathbb{D}
$$


and there is a cyclic symmetry of the actions $d_{1}, d_{2}, d_{3}$. The global action of the $\mathrm{SO}(8)$ subgroup on $J_{3}^{8}$ can then be written as:

$$
\mathrm{SO}(8): J \rightarrow\left(\begin{array}{ccc}
\frac{\alpha_{1}}{\left(d_{3} O_{3}\right)} & \left(d_{3} O_{3}\right) & \overline{\left(d_{2} O_{2}\right)} \\
\left(d_{2} O_{2}\right) & \frac{\alpha_{2}}{\left(d_{1} O_{1}\right)} & \left.\alpha_{3} O_{1}\right)
\end{array}\right) .
$$

The infinitesimal generators of $\mathrm{SO}(8)$ satisfy the principle of local triality (sometimes called infinitesimal triality):

$$
\left(D_{1} O_{1}\right) O_{2}+O_{1}\left(D_{2} O_{2}\right)=\overline{D_{3} \overline{\left(O_{1} O_{2}\right)}},
$$

where $D_{i}$ belongs to the Lie algebra of $\mathrm{SO}(8)$. An example of triality action, which we shall use later, is given by

$$
d_{1}=L_{a} \quad d_{2}=R_{a} \quad d_{3}=L_{\bar{a}} R_{\bar{a}},
$$

where $a$ is a unit octonion and $L_{a}$ and $R_{a}$ stand for multiplication from the left and the right by the octonion $a$.

We now prove four lemmas which we shall need in the following [22]. They all apply to one-dimensional projection operator $P$.

Lemma 1. There exists always a transformation belonging to $F_{4}$ which brings $P$ given by (2.14) to the form $E_{1}$ (see (3.6)).

Lemma 2. Given $P_{1}, P_{2}, P_{3}$ such that $P_{1} \circ P_{2}=P_{2} \circ P_{3}=P_{3} \circ P_{1}=0$ then there exists always a transformation of $F_{4}$ which brings them to the form $E_{1}, E_{2}, E_{3}$.

Lemma 3. Given any two $P_{1}$ and $P_{2}$ then there exists always a transformation of $F_{4}$ which brings them into a real form.

Lemma 4. $\operatorname{tr} P_{1} \circ P_{2}=0$ implies $P_{1} \circ P_{2}=0$. For the proof of Lemma 1, we start with the general form (2.14) for $P$. Consider the $\mathrm{SO}(9)$ rotation

$$
\begin{aligned}
R_{12}(\varphi) & =\exp \left(\varphi D_{(x)_{12}, E_{1}-E_{2}}\right) \\
x & =\frac{a \bar{b}}{[n(a \bar{b})]^{1 / 2}} \\
\sin \varphi & =\frac{2[n(a \bar{b})]^{1 / 2}}{n(a)+n(b)} \quad \cos \varphi=\frac{n(a)-n(b)}{n(a)+n(b)} .
\end{aligned}
$$

One then gets, using (3.3)

$$
P^{\prime}=R_{12} P=(n(a)+n(b)) E_{1}+n(c) E_{3}+\left(\frac{n(a)+n(b)}{n(a)}\right)^{1 / 2}(c \bar{a})_{31} .
$$

Now, perform the $\mathrm{SO}(9)$ rotation

$$
\begin{aligned}
R_{31}(\psi) & =\exp \left(\psi D_{(y)_{31}, E_{3}-E_{1}}\right) \\
y & =\frac{c \bar{a}}{[n(c \bar{a})]^{1 / 2}} \\
\sin \psi & =-2[n(c)(n(a)+n(b))]^{1 / 2} /[n(a)+n(b)+n(c)]^{1 / 2} \\
\cos \psi & =-[n(c)-n(a)-n(b)] /[n(a)+n(b)+n(c)]^{1 / 2}
\end{aligned}
$$


The result is

$$
P^{\prime \prime}=R_{31} P^{\prime}=R_{31} R_{12} P=E_{1} .
$$

With an appropriate permutation of indices and letters one could also get $E_{2}$ and $E_{3}$.

To prove Lemma 2 we first bring $P_{1}$ to the form $E_{1}$ leaving $P_{2}$ in the general form (2.14). Then

$$
E_{1} \circ P_{2}=a \bar{a} E_{1}+\frac{1}{2}(a \bar{b})_{12}+\frac{1}{2}(c \bar{a})_{31} .
$$

$E_{1} \circ P_{2}=0$ implies $a=0$, hence the most general form for $P_{2}$ is $P_{2}=b \bar{b} E_{2}+c \bar{c} E_{3}+(b \bar{c})_{23}$ with $b \bar{b}+c \bar{c}=1$. The transformation $R_{23}(\varphi)$ obtained from (3.12) by the cyclic permutation $1 \rightarrow 2 \rightarrow 3$ and $a \rightarrow b \rightarrow c$ brings $P_{2}$ to the form $E_{2}$, while leaving $E_{3}$ invariant. It is then obvious that the only $P_{3}$ that satisfies $P_{3} \circ E_{1}=P_{3} \circ E_{2}=0$ is $P_{3}=E_{3}$.

For Lemma 3 we bring $P_{1}$ to the form $E_{3}$ with $P_{2}$ given by (2.14). The SO(9) rotation $R_{12}(\varphi)$ given by (3.12) brings $P_{2}$ to the form (3.13). An $\mathrm{SO}(8)$ rotation via the triality action given by (3.11):

$$
d_{1}=L_{O} \quad d_{2}=R_{O} \quad d_{3}=L_{O} R_{O}
$$

with $O$ chosen as $\frac{a \bar{c}}{|n(a \bar{c})|^{1 / 2}}$ brings $P_{2}$ into a real form.

For Lemma 4, first consider an $F_{4}$ transformation that brings $P_{1}$ into the form $E_{1}$ with $P_{2}$ being of the general form (2.14). Then from (3.16) we have

$$
\operatorname{tr} E_{1} \circ P_{2}=a \bar{a}
$$

therefore $\operatorname{tr} E_{1} \circ P_{2}=0$ implies $a=0$ and thus $E_{1} \circ P_{2}=0$.

Lemma 5. Any element of $J_{3}^{8}$ can be brought to the diagonal form by an $F_{4}$ transformation.

Consider a general element $X$ of $J_{3}^{8}$ as given by (3.6) then by the triality action

$$
d_{1}=L \frac{x_{3}}{\left|x_{3}\right|} R \frac{x_{3}}{\left|x_{3}\right|} \quad d_{2}=L \frac{\overline{x_{3}}}{\left|x_{3}\right|} \quad d_{3}=R \frac{\overline{x_{3}}}{\left|x_{3}\right|} .
$$

it is brought to the form

$$
X_{1}=\left(\begin{array}{ccc}
\alpha_{1} & \left|x_{3}\right| & \bar{x}_{2}^{\prime} \\
\left|x_{3}\right| & \alpha_{2} & x_{1}^{\prime} \\
x_{2}^{\prime} & \bar{x}_{1}^{\prime} & \alpha_{3}
\end{array}\right) \text {. }
$$

Then by a simple real rotation this can be brought to the form

$$
X_{2}=\left(\begin{array}{ccc}
\alpha_{1}^{\prime} & 0 & \bar{x}_{2}^{\prime \prime} \\
0 & \alpha_{2} & x_{1}^{\prime \prime} \\
x_{2}^{\prime \prime} & x_{1}^{\prime \prime} & \alpha_{3}
\end{array}\right)
$$

Again via the triality action this can be brought to the form

$$
X_{3}=\left(\begin{array}{ccc}
\alpha_{1}^{\prime} & 0 & \bar{x}_{2}^{\prime \prime \prime} \\
0 & \alpha_{2}^{\prime \prime} & \left|x_{1}^{\prime \prime}\right| \\
x_{2}^{\prime \prime \prime} & \left|x_{1}^{\prime \prime}\right| & \alpha_{3}^{\prime}
\end{array}\right)
$$


Now under the rotation

$$
T X_{3} T^{-1}=X_{4} \text {, }
$$

where $T=\left(\begin{array}{ccc}1 & 0 & 0 \\ 0 & \mathrm{R}\end{array}\right)$, with $R$ being the real rotation matrix that diagonalizes $\left(\begin{array}{cc}\alpha_{2}^{\prime \prime} & \left|x_{1}^{\prime \prime}\right| \\ \left|x_{1}^{\prime \prime}\right| & \alpha_{3}^{\prime}\end{array}\right)$, we find

$$
X_{4}=\left(\begin{array}{ccc}
\alpha_{1}^{\prime} & -\sin \alpha \bar{x}_{2}^{\prime \prime \prime} & \cos \alpha \bar{x}_{2}^{\prime \prime \prime} \\
-\sin \alpha x_{2}^{\prime \prime \prime} & \alpha_{2}^{\prime \prime \prime} & 0 \\
\cos \alpha x_{2}^{\prime \prime \prime} & 0 & \alpha_{3}^{\prime \prime}
\end{array}\right)
$$

Then by the triality action

$$
d_{3}=R \frac{x_{2}^{\prime \prime \prime}}{\left|x_{2}^{\prime \prime \prime}\right|} \quad d_{2}=L \frac{\bar{x}_{2}^{\prime \prime \prime}}{\left|x_{2}^{\prime \prime \prime}\right|} \quad d_{1}=L \frac{\bar{x}_{2}^{\prime \prime \prime}}{\left|x_{2}^{\prime \prime \prime}\right|} R \frac{x_{2}^{\prime \prime \prime}}{\left|x_{2}^{\prime \prime \prime}\right|} .
$$

$X_{4}$ is brought to a real symmetric matrix which can always be diagonalized by a real rotation matrix. QED.

\section{The Moufang Projective Plane}

The representation theorem for projective spaces states that projective spaces of dimension $n>2$ can be represented by vector spaces over a skew field [23]. If the underlying division ring is commutative then Pappus-Pascal-theorem holds. The Desargues' theorem follows from the projective axioms for $n>2$.

One example of a non-Desarguian projective plane $(n=2)$ was given by Moufang [16]. She gave an affine coordination of this geometry in terms of octonions and showed that the harmonic lock incidence theorem (which is a weaker theorem than Desargues' theorem) is satisfied as a consequence of the alternativity of the underlying octonion algebra.

Jordan gave a construction of the Moufang plane in terms of the idempotents of $J_{3}^{8}[17]$. The first rigorous study of the Moufang plane and its automorphism group was given by Freudenthal [24]. Below we shall use Jordan's method in our study of the Moufang plane and follow the work of Freudenthal [20], Springer [25], and the excellent review of Jacobson [3].

A projective plane is defined as a set of elements called points and a collection of subsets of points called lines such that the three following axioms are satisfied. $G=$ Moufang plane

1) Any two distinct points are contained in one and only one line.

2) The intersection of any two distinct lines is one point.

3) There exist four points no three of which are in the same line: as an immediate consequence, we can prove

4) Given three distinct lines which intersect in three points, the line defined by two other points of two of these lines intersects the third one.

5) Any line contains at least three points. 
If we take 1), 4), and 5) as axioms we define an irreducible projective geometry. As a matter of fact, by 2) such a projective geometry is a plane. A linear variety is a subset of points such that, each time it contains two points, it contains the line defined by this two points. Here, for the projective plane the linear varieties are the void set, the points, the lines and plane itself.

$\mathrm{Up}$ to a real factor, one-dimensional projection operators (or irreducible idempotents) are given by the condition

$$
P \times P=0 .
$$

As points in the plane we shall take the equivalence class $P$ satisfying (4.1) and as a representative of this class take a $P$ satisfying the trace condition

$$
\operatorname{tr} P=1 .
$$

A line in this plane will be represented by a two-dimensional projection operator $l$

$$
l^{2}=l ; \quad t r l=2
$$

A point $P$ is said to be contained in the line $l$ if and only if

$$
P \circ l=P \quad \text { or } \quad P \circ(I-l)=0 .
$$

To prove that axioms 1,2 , and 3 are satisfied, one first needs to show that for $P_{1}$ and $P_{2}$ different one-dimensional projection operators,

$$
L_{12}=\frac{P_{1} \times P_{2}}{\operatorname{tr} P_{1} \times P_{2}}
$$

is a one-dimensional projection operator. This can be simply done by considering the case $P_{1}=E_{1}$ and $P_{2}$ general and extending it to the general case. Or else, one can use the identity

$$
\begin{aligned}
& 2\left(J_{1} \times J_{1}\right) \times\left(J_{2} \times J_{2}\right)+4\left(J_{1} \times J_{2}\right) \times\left(J_{1} \times J_{2}\right) \\
& \quad=\left(J_{1} \times J_{1}, J_{2}\right) J_{2}+\left(J_{1}, J_{2} \times J_{2}\right) J_{1} .
\end{aligned}
$$

It then follows that

$$
l_{12}=I-L_{12}=I-\frac{P_{1} \times P_{2}}{\operatorname{tr} P_{1} \times P_{2}}
$$

is a two-dimensional projection operator and hence a line. The points $P_{1}$ and $P_{2}$ lay on the line $l_{12}$ since

$$
\left(P_{1}, L_{12}\right)=\frac{1}{\operatorname{tr} P_{1} \times P_{2}}\left(P_{1}, P_{1} \times P_{2}\right)=\frac{1}{\operatorname{tr} P_{1} \times P_{2}}\left(P_{1} \times P_{1}, P_{2}\right)=0
$$

and

$$
\left(P_{2}, L_{12}\right)=\frac{1}{\operatorname{tr} P_{1} \times P_{2}}\left(P_{2}, P_{1} \times P_{2}\right)=\frac{1}{\operatorname{tr} P_{1} \times P_{2}}\left(P_{2} \times P_{2}, P_{1}\right)=0 .
$$

To show that $l_{12}$ is the only line through $P_{1}$ and $P_{2}$, one needs: 
Lemma 6. If $P_{1}$ and $P_{2}$ are different one-dimensional projectors then the projector $P_{3}$ satisfies $P_{1} \circ P_{3}=P_{2} \circ P_{3}=0$ if and only if $P_{3}$ is a multiple of $P_{1} \times P_{2}$.

It is enough to prove the lemma for $P_{3}=E_{1}, P_{1}$ and $P_{2}$ being of the general form (2.14). Then $P_{i} \circ P_{3}=0(i=1,2)$ implies

$$
P_{i}=b_{i} \bar{b}_{i} E_{2}+c_{i} \bar{c}_{i} E_{3}+\left(b_{i} \bar{c}_{i}\right)_{23} \text {. }
$$

Direct computation gives $P_{1} \times P_{2}$ proportional to $E_{1}$. The converse has already been proven above. Therefore, axiom 1 is satisfied.

The intersection of two lines $I-P_{1}$ and $I-P_{2}$ is the point

$$
P_{12}=\frac{P_{1} \times P_{2}}{\operatorname{tr} P_{1} \times P_{2}}
$$

which, by the same arguments, can be shown to be unique. Hence, axiom 2 is satisfied. To prove axiom 3, consider the following four points

$$
P_{i}=E_{i} \quad i=1,2,3
$$

with $P_{4}$ being given by the general formula (2.14). The line $l_{K}$ passing through points $E_{i}$ and $E_{j}$ is given by

$$
\frac{E_{i} \times E_{j}}{\operatorname{tr} E_{i} \times E_{j}}=E_{k}=I-l_{k} ; i, j, k \quad \text { in cyclic order } .
$$

The points $E_{k}$ and $P_{4}$ do not lay on the line $l_{k}$ since

$$
E_{k} \circ E_{k} \neq 0 ; \quad E_{k} \circ P_{4} \neq 0 .
$$

Thus, there exist four points, no three of which lay on the same line. Such a projective plane constructed with Jordan matrices is called a Moufang plane.

Let us now show that this projective plane is effectively non-Desarguian. First let us recall that Desargues' theorem states that given two triangles $A B C$ and $A^{\prime} B^{\prime} C^{\prime}$ such that the lines $A A^{\prime}, B B^{\prime}$, and $C C^{\prime}$ intersect at a common point 0 , then the intersection points of the lines $A B$ and $A^{\prime} B^{\prime}, B C$ and $B^{\prime} C^{\prime}, A C$ and $A^{\prime} C^{\prime}$ all lay on a straight line.

We now give an explicit non-Desarguian configuration. For this consider the three lines

$$
\begin{aligned}
l_{3} & =I-E_{3} ; \quad l_{2}=I-E_{2} ; \quad l_{\theta}=I-\theta_{23} \\
E_{3} & =\left(\begin{array}{lll}
0 & 0 & 0 \\
0 & 0 & 0 \\
0 & 0 & 1
\end{array}\right) ; \quad E_{2}=\left(\begin{array}{lll}
0 & 0 & 0 \\
0 & 1 & 0 \\
0 & 0 & 0
\end{array}\right) \\
\theta_{23} & =\left(\begin{array}{ccc}
0 & 0 & 0 \\
0 & \sin ^{2} \theta & -\sin \theta \cos \theta \\
0 & -\sin \theta \cos \theta & \cos ^{2} \theta
\end{array}\right) .
\end{aligned}
$$


These three lines intersect at the point represented by the idempotent $E_{1}$ since

$$
\begin{aligned}
& E_{2} \times E_{3}=\frac{1}{2} E_{1} \\
& E_{2} \times \theta_{23}=\frac{\cos ^{2} \theta}{2} E_{1} \\
& E_{3} \times \theta_{23}=\frac{\sin ^{2} \theta}{2} E_{1} .
\end{aligned}
$$

Now take two points on each line

$$
\begin{aligned}
& P_{A}, P_{A^{\prime}} \in l_{3} \\
& Q_{B}, Q_{B^{\prime}} \in l_{2} \\
& R_{C}, R_{C^{\prime}} \in l_{\theta} .
\end{aligned}
$$

Graphically, we have

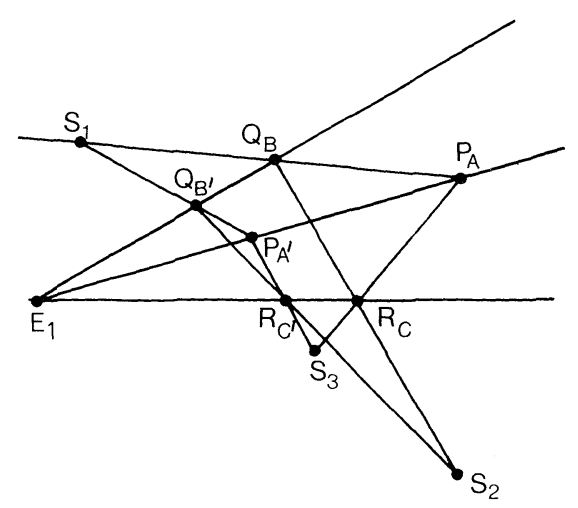

The line passing through points $P_{A}$ and $Q_{B}$ is represented by $I-L_{P Q}$; $L_{P Q}=P_{A} \times Q_{B} / \operatorname{tr} P_{A} \times Q_{B}$ and intersection point of the lines $l_{P Q}$ and $l_{P^{\prime} Q^{\prime}}$ is the point (up to a trace factor)

$$
S_{1}=\left(P_{A} \times Q_{B}\right) \times\left(P_{A^{\prime}} \times Q_{B^{\prime}}\right) .
$$

Similarly for the points $B, C, B^{\prime}, C^{\prime}$ and $A, C, A^{\prime}, C^{\prime}$ one finds

$$
\begin{aligned}
& S_{2}=\left(Q_{B} \times R_{C}\right) \times\left(Q_{B^{\prime}} \times R_{C^{\prime}}\right) \\
& S_{3}=\left(P_{A} \times R_{C}\right) \times\left(P_{A^{\prime}} \times R_{C^{\prime}}\right) .
\end{aligned}
$$

Now Desargues' theorem would require that the points $S_{1}, S_{2}$, and $S_{3}$ lay on a straight line, which in our notation is equivalent to the condition

$$
\left(S_{1} \times S_{2}, S_{3}\right)=\left[S_{1}, S_{2}, S_{3}\right]=0 .
$$


Let us now show that this condition does not in general hold for this Moufang plane. As the points in our plane consider the idempotents

$$
\begin{aligned}
P_{A} & =\frac{1}{2}\left(\begin{array}{ccc}
1 & -e_{2} & 0 \\
e_{2} & 1 & 0 \\
0 & 0 & 0
\end{array}\right) \quad P_{A^{\prime}}=\frac{1}{2}\left(\begin{array}{ccc}
1 & -e_{1} & 0 \\
e_{1} & 1 & 0 \\
0 & 0 & 0
\end{array}\right) \\
Q_{B} & =\frac{1}{2}\left(\begin{array}{ccc}
1 & 0 & e_{7} \\
0 & 0 & 0 \\
-e_{7} & 0 & 1
\end{array}\right) \quad Q_{B^{\prime}}=\frac{1}{2}\left(\begin{array}{ccc}
1 & 0 & e_{3} \\
0 & 0 & 0 \\
-e_{3} & 0 & 1
\end{array}\right) \\
R_{C} & =\frac{1}{2}\left(\begin{array}{ccc}
1 & -\cos \theta e_{6} & -\sin \theta e_{6} \\
\cos \theta e_{6} & \cos { }^{2} \theta & \cos \theta \sin \theta \\
\sin \theta e_{6} & \cos \theta \sin \theta & \sin 2 \theta
\end{array}\right) \\
R_{C^{\prime}} & =\frac{1}{2}\left(\begin{array}{ccc}
1 & -\cos \theta e_{5} & -\sin \theta e_{5} \\
\cos \theta e_{5} & \cos { }^{2} \theta & \cos \theta \sin \theta \\
\sin \theta e_{5} & \cos \theta \sin \theta & \sin 2 \theta
\end{array}\right) .
\end{aligned}
$$

After some long and tedious calculation, one finds that

$$
\left(S_{1} \times S_{2}, S_{3}\right)=\frac{1}{2^{19}} \cos ^{2} \theta \sin ^{2} \theta(3+\sin \theta-\cos \theta+\sin \theta \cos \theta) .
$$

This shows the existence of non-Desargian configurations in the Moufang plane.

\section{Orthocomplementation of the Moufang Plane}

A projective plane is orthocomplemented if on it there exists an involution on the linear varieties which reverses the order of inclusion and maps each point to a line outside this point. Existence of such involutions over the Moufang plane has long been established [26] and thoroughly studied [27]. The simplest such involution is to associate with each projector $P$ the projector

$$
P^{\prime}=I-P \text {. }
$$

If $P$ is one dimensional $(\operatorname{tr} P=1$ ), then $I-P$ is of dimension 2 and represents a line. This involution reverses the order of inclusion since if $P \circ l=P$ then

$$
P^{\prime} \circ l^{\prime}=(I-P) \circ(I-l)=I-l-P+P=l^{\prime} .
$$

A linear variety is by definition orthogonal to another if the orthocomplement of one contains the other. Two points $P_{1}$ and $P_{2}$ are orthogonal if and only if $P_{1} \circ P_{2}=0$

$$
P_{1} \circ P_{2}=0 \Leftrightarrow P_{1} \perp P_{2} \text {. }
$$

In this case

$$
P_{1} \times P_{2}=\frac{1}{2}\left(I-P_{1}-P_{2}\right)
$$


and

$$
l_{12}=P_{1}+P_{2} .
$$

The third point orthogonal to $P_{1}$ and $P_{2}$ is just $\left(I-P_{1}-P_{2}\right)$, which is the orthocomplement of the line $l_{12}$. We remark that for any orthogonal linear varieties $l_{1}$ and $l_{2}$ we have the simple formula for their union

$$
l_{1} \cup l_{2}=l_{1}+l_{2} \text {. }
$$

Having now at our disposal an orthocomplemented projective geometry, we can construct a quantum mechanical system of propositions, satisfying all axioms, as shown in Ref. [15]. As in [15], two propositions $l_{1}$ and $l_{2}$ are compatible if and only if $\left(l_{1} \cap l_{2}\right) \cup\left(l_{1} \cap l_{2}^{\prime}\right)=l_{1}$ i.e. if $l_{1}$ can be written as the sum of two projectors, one in $l_{2}$ and one in $l_{2}^{\prime}$

$$
\begin{aligned}
& l_{1}=P_{1}+P_{2} \quad \text { with } \quad P_{1} \circ l_{2}=P_{1} \\
& P_{2} \circ l_{2}=0 .
\end{aligned}
$$

In $\S 6$ we show the existence of a unique probability function and in $\S 7$ the theory of measurements and observables is discussed.

The automorphism group of the orthocomplemented Moufang plane is again $F_{4}$ [3]. Indeed, since $F_{4}$ preserves the Jordan product and the Freudenthal product, $F_{4}$ transforms points into points, lines into lines and orthogonal linear varieties into orthogonal linear varieties.

\section{The Probability Function}

We now show that given any one-dimensional projector $P$, there exists a unique probability function

$$
W_{p}(l)=\operatorname{tr}(P \circ l) \quad l \in G
$$

satisfying Gleason's axioms [28]

1) $0 \leqq W_{p}(l) \leqq 1$

2) $W_{p}(P)=1$

3) $W_{p}\left(l_{1} \vee l_{2}\right)=W_{p}\left(l_{1}\right)+W_{p}\left(l_{2}\right)$

$$
l_{1} \perp l_{2} \in G \text {. }
$$

To prove (6.2), we first choose for $l$ a point $Q$. Since $\operatorname{tr} P \circ Q$ is invariant under $F_{4}$, we consider $P$ in the form $E_{1}$ and $Q$ general given by (2.14). Then $\operatorname{tr} P \circ Q$ $=|a|^{2} \leqq 1$. Next, for $l=I-Q, \operatorname{tr} P \circ l=1-|a|^{2} \geqq 0$.

To prove (6.4), we remark that according to (5.5), $W_{p}\left(l_{1} \cup l_{2}\right)=\operatorname{tr}\left(P \circ\left(l_{1}+l_{2}\right)\right)$ $=\operatorname{tr}\left(P l_{1}\right)+\operatorname{tr}\left(P l_{2}\right)=W_{p}\left(l_{1}\right)+W_{p}\left(l_{2}\right)$. On the other hand, such a function is unique i.e. if a real function on $G$ satisfies (6.2)-(6.4) it is identical with the one given by the trace. To prove this, let us first make two remarks

1) Due to the condition (6.4) the function $W_{p}(l)$ is completely defined by its values on the points. 
2) The restriction of $W_{p}$ to the real subplane defined by the real $3 \times 3$ projection operators is a state on this plane and by Gleason theorem it is unique and given by the trace. This is indeed the case since the orthogonality condition $P \circ Q=0$ (Jordan product) restricted to real $3 \times 3$ projectors is identical with $P Q=0$ (ordinary matrix product).

Suppose there exists a point $Q$ such that $W_{p}(Q)$ is different from $\operatorname{tr}(P Q)$. Lemma 3 of $\S 3$ shows that by an automorphism of $F_{4}$, we can choose $P$ and $Q$ real. Then by the second remark, we will have a contradiction and by the first remark the uniqueness is proven.

\section{Measurements and Observables}

We consider here ideal measurements of the first kind [15] which are simply called measurements in usual text-books. We show that the result of two successive, compatible experiments does not depend on the order in which they are performed, in spite of the non-associativity of octonions.

We first consider a physical system in the state $P$ (one-dimensional projection operator) and we measure the proposition $l$ (yes-no experiment). Suppose the result of the experiment is the answer yes (the value one). What is then the final state after the measurement? The problem is only non-trivial if $l$ is a line. According to reference [29], the final state is the point

$$
P_{f}=\left(P \cup l^{\prime}\right) \cap l,
$$

where $l^{\prime}$, the orthocomplement of $l$, is a point. $P \cup l^{\prime}$ is the line

$$
\hat{l}=I-\frac{P \times l^{\prime}}{\operatorname{tr} P \times l^{\prime}}=I-\hat{L} .
$$

Then the intersection of the lines $l$ and $\hat{l}$ is the point $l^{\prime} \times \hat{L}$. Normalizing, we get the final state

$$
P_{f}=\frac{l^{\prime} \times \hat{L}}{\operatorname{tr}\left(l^{\prime} \times \hat{L}\right)}=\frac{l^{\prime} \times\left(P \times l^{\prime}\right)}{\operatorname{tr}\left(l^{\prime} \times\left(P \times l^{\prime}\right)\right)} .
$$

Using Equation (2.16) and the fact that the free Jordan algebra with two generators (here $P$ and $l^{\prime}$ ) is special, i.e. equivalent to a matrix algebra over an associative field with Jordan product [4], one finds

$$
\begin{aligned}
\operatorname{tr}\left(\left(P \circ l^{\prime}\right) \circ l^{\prime}\right) & =\operatorname{tr} P \circ l^{\prime}, \\
\operatorname{tr}\left(\left(P \times l^{\prime}\right) \times l^{\prime}\right) & =\frac{1}{4}\left(1-\operatorname{tr} P \circ l^{\prime}\right), \\
\left(P \times l^{\prime}\right) \times l^{\prime} & =\left(P \circ l^{\prime}\right)\left(l^{\prime}-I\right)+\frac{1}{4}\left(P-l^{\prime} \operatorname{tr} P \circ l^{\prime}\right) .
\end{aligned}
$$

The probability to get the answer yes if one measures the proposition $l$ on a system in state $P$ is

$$
W(l)=\operatorname{tr} P \circ l .
$$


Suppose now we measure $l_{1}$ on the initial state $P_{1}$ and get the state $P_{l_{1}}$. Then we measure the compatible [see Eq. (5.6)] observable $l_{2}$. The probability to get the answer yes for $l_{1}$ and yes for $l_{2}$ is $\operatorname{tr}\left(P_{l_{1}} \circ l_{2}\right) \operatorname{tr}\left(P \circ l_{1}\right)$. Therefore, we must have

$$
\operatorname{tr}\left(P_{l_{1}} \circ l_{2}\right) \operatorname{tr}\left(P \circ l_{1}\right)=\operatorname{tr}\left(P_{l_{2}} \circ l_{1}\right) \operatorname{tr}\left(P \circ l_{2}\right) .
$$

From Equation (7.3) we have

$$
P_{l_{1}}=4\left(1-\operatorname{tr} P l_{1}^{\prime}\right)^{-1}\left(P \times l_{1}^{\prime}\right) \times l_{1}^{\prime},
$$

where $l_{1}^{\prime}=I-l_{1}$.

Then

$$
\begin{aligned}
\operatorname{tr} P_{l_{1}} \circ l_{2} & =4\left(1-\operatorname{tr} P l_{1}^{\prime}\right)^{-1} \operatorname{tr}\left[\left(\left(P \times l_{1}^{\prime}\right) \times l_{1}^{\prime}\right) \circ l_{2}\right] \\
& =1-4\left(1-\operatorname{tr} P l_{1}^{\prime}\right)^{-1} \operatorname{tr}\left[\left(P \times l_{1}^{\prime}\right) \circ\left(l_{1}^{\prime} \times l_{2}^{\prime}\right)\right] \\
& =1-4\left(1-\operatorname{tr} P l_{1}^{\prime}\right)^{-1} \operatorname{tr}\left[P \circ\left(l_{1}^{\prime} \times\left(l_{1}^{\prime} \times l_{2}^{\prime}\right)\right)\right] .
\end{aligned}
$$

The compatibility of $l_{1}$ and $l_{2}$ implies $l_{1}^{\prime} \circ l_{2}^{\prime}=0$, hence

$$
\begin{gathered}
l_{1}^{\prime} \times\left(l_{1}^{\prime} \times l_{2}^{\prime}\right)=\frac{1}{4} l_{2}^{\prime} \\
\operatorname{tr} P \circ\left(l_{1}^{\prime} \times\left(l_{1}^{\prime} \times l_{2}^{\prime}\right)\right)=\frac{1}{4} \operatorname{tr} P \circ l_{2}^{\prime} .
\end{gathered}
$$

Thus, we find

$$
\begin{aligned}
\operatorname{tr} P_{l_{1}} \circ l_{2} & =1-\left(1-\operatorname{tr} P \circ l_{1}^{\prime}\right)^{-1} \operatorname{tr} P \circ l_{2}^{\prime} \\
& =\left(1-\operatorname{tr} P \circ l_{1}^{\prime}\right)^{-1}\left(1-\operatorname{tr} P \circ l_{1}^{\prime}-\operatorname{tr} P \circ l_{2}^{\prime}\right) .
\end{aligned}
$$

Since $\operatorname{tr}\left(P l_{1}\right)=\operatorname{tr} P \circ\left(I-l_{1}^{\prime}\right)=1-\operatorname{tr} P l_{1}^{\prime}$, we get the result

$$
\operatorname{tr}\left(P_{l_{1}} \circ l_{2}\right) \operatorname{tr}\left(P \circ l_{1}\right)=1-\operatorname{tr} P \circ l_{1}^{\prime}-\operatorname{tr} P \circ l_{2}^{\prime}=2 \operatorname{tr} P \circ\left(l_{1}^{\prime} \times l_{2}^{\prime}\right) .
$$

Since the right-hand-side is symmetric in $l_{1}^{\prime}$ and $l_{2}^{\prime}$, we have

$$
\operatorname{tr}\left(P_{l_{1}} \circ l_{2}\right) \operatorname{tr}\left(P \circ l_{1}\right)=\operatorname{tr}\left(P_{l_{2}} \circ l_{2}\right) \operatorname{tr}\left(P \circ l_{2}\right)=2 \operatorname{tr} P \circ\left(l_{1}^{\prime} \times l_{2}^{\prime}\right) .
$$

Note that the point $2 l_{1}^{\prime} \times l_{2}^{\prime}$ is just the intersection of the lines $l_{1}$ and $l_{2}$, as expected.

What (7.11) implies is that the probability of successive, compatible experiments is independent of the order of the measurements. This is a somewhat surprizing result in view of the nonassociative nature of the octonion product.

We now come to the question of observables [30]. First, each projection operator defines an observable. In general, in the Moufang plane, an observable is defined by three one-dimensional projections. They can always be brought by an $F_{4}$-transformation to the form $E_{1}, E_{2}$, and $E_{3}$ (Lemma 2). The question arises if any Jordan matrix can be written as a linear superposition of three mutually orthogonal one-dimensional projection operators and interpreted as an observable. The answer is yes, since any element of $J_{3}^{8}$ can be diagonalized by an $F_{4}$ transformation (Lemma 5).

In order to get a Schrödinger equation, we need a time evolution operator. This should transform orthogonal states into orthogonal states and hence belong 
to the automorphism group of the orthocomplemented Moufang plane, which is $F_{4}$. Some generator in the Lie algebra of $F_{4}$ will play the role of the Hamiltonian.

The subalgebra of complex Jordan matrices can be used to construct a Desarguian projective subgeometry of the Moufang geometry. The corresponding Quantum Mechanics can be realized in a three-dimensional Hilbert space. The subgroup of $F_{4}$ which leaves the complex subgeometry invariant is $\mathrm{SU}(3)^{c}$. Jordan matrices whose elements comprise the six remaining octonionic units transform as 3 and $\overline{3}$ under $\mathrm{SU}(3)^{c}$. More precisely, under the maximal subgroup $\mathrm{SU}(3)^{c} \times \mathrm{SU}(3)$ the representation $\underline{26}$ of $F_{4}$ reduces as

$$
\underline{26}=\left(3^{c}, 3\right) \oplus\left(\overline{3}^{c}, \overline{3}\right) \oplus\left(1^{c}, 8\right) .
$$

Hence, it is tempting to identify $\mathrm{SU}(3)^{c}$ with the color group, complex Jordan matrices with lepton states $[18,31]$, and the other with quark and antiquark states (note that a projection operator belongs to the reducible representation $27=26 \oplus 1$ ). Since a non-Desarguian projective plane cannot be embedded in a irreducible projective geometry of higher dimension, this would mean that quarks have no space properties, these latter requiring an infinite dimensional geometry. On the other hand, the lepton subgeometry can hopefully be embedded in a consistent way in such an infinite geometry. However, the definition of a consistent tensor product of quark states is still an open problem.

\section{References}

1. Jordan,P.: Nachr. Ges. Wiss. Göttingen 569-575 (1932); 209-214 (1933); Z. Physik 80, 285 (1933)

2. Jordan,P., Neumann,J.von, Wigner, E. : Ann. Math. 36, 29-64 (1934)

3. Jacobson, N.: Structure and representations of Jordan algebras. Am. Math. Soc. Coll. Publ. 39, $356-411(1968)$

4. Schafer, R.D.: An introduction to non-associative algebras. New York-London: Academic Press 1966

5. Pais, A.: Phys. Rev. Letters 7, 291 (1961)

6. Gamba, A.: High energy physics and elementary particles (ed. A. Salam). Vienna: IAEA 1965

7. Goldstine,H.H., Horwitz,L.P.: Proc. Nat. Acad. 48, 1134 (1962); Math. Ann. 154, 1 (1964);

Horwitz,L.P., Biedenharn,L.C.: Helv. Phys. Acta 38, 385 (1965); Exceptional parafermions in a Hilbert space over an associative algebra. Preprint (1977)

Buccella,F., Falcioni,M., Pugliese, A.: Lett. Nuovo Cimento 18, 441-446 (1977)

8. Günaydin,M., Gürsey,F.: Lett. Nuovo Cimento 6, 401 (1973)

9. Günaydin, M., Gürsey,F.: J. Math. Phys. 14, 1651 (1973)

10. Günaydin, M.: Ph. D. Thesis, Yale University (1973) (unpublished)

11. Günaydin,M., Gürsey,F.: Phys. Rev. D9, 3387 (1974)

12. Gürsey,F.: Johns Hopkins University workshop on current problems in high energy particle theory. Baltimore, Md. (1974)

13. Günaydin, M. : J. Math. Phys. 17, 1875 (1976)

14. Fritzsch,H., Gell-Mann, M.: Proceedings of the XVI. International Conference on High Energy Physics, Chicago-Batavia. Ill. (1972). (ed. J. D. Jackson. A. Roberts), Vol. 2, p. 135. Batavia, Ill.: NAL 1973

15. Piron,C.: Foundations of quantum physics. Reading, Mass.: Benjamin 1976

Emch, G. : Algebraic methods in statistical mechanics and quantum field theory. New York: WileyInterscience 1972

16. Moufang, R.: Abh. Math. Sem. Univ. Hamburg 9, 207-222 (1933)

17. Jordan,P.: Abh. Math. Sem. Univ. Hamburg 16, 74-76 (1949)

18. Gürsey,F.: Yale Report 400-3075-178 
19. This is one of the four possible bilinear products that can be defined over the octonions, see [13]

20. Freudenthal,H.: Advan. Math. 1, 145-190 (1964)

21. For the literature on the principle of triality, we refer the reader to [4]

Yokota,I.: Below, we shall follow: J. Fac. Sci. Shinshu University 3, 35 (1968)

22. Below, we shall give simple proofs of these lemmas. For more sophisticated and general treatment of related problems see, for example:

Springer,T.A., Veldkamp,F.D.: Proc. Koninkl. Akad. Wetenschap A66, 413-451 (1963)

23. Bumcrot,R.J.: Modern projective geometry, Chapter III. New York: Halt, Rinehart, Winston 1969

24. Freudenthal,H.: Oktaven, Ausnahmegruppen und Oktavengeometrie. Utrecht (mimeographed) (1951)

25. Springer, T. A.: Proc. Koninkl. Akad. Wetenschap A63, 74-101 (1960)

26. Tits,J.: Bull. Acad. Roy. Belg. Sci. 39, 309-329 (1953)

27. Freudenthal,H.: Proc. Koninkl. Ned. Akad. Wetenschap, A58, 151-157 (1955); A62, 165-201 (1959)

28. Gleason, A. M.: J. Math. Mech. 6, 885 (1957)

29. See [15], p. 68

30. See $[15], \S 2.4$

31. Gürsey,F.: New pathways in high energy physics, I (ed. A.Perlmatter). New York: Plenum Press 1976

Communicated by R. Haag

Received December 20, 1977 
